\title{
Análisis a la producción científica en auditoría de recursos humanos
}

\author{
Scientific production analysis \\ at human resources auditing
}

\author{
Jorge Sánchez Henríquez ${ }^{1}$, Ignacio Yáñez Vidal ${ }^{2}$
}

\begin{abstract}
RESUMEN
El propósito del presente artículo es realizar una revisión de la producción científica relacionada Auditoria de Recursos Humanos en la base de datos de SciELO, considerándola como eslabón inicial en términos de calidad frente a bases de datos de talla mundial como Scopus y Web of Science, ocupando las técnicas que otorga la bibliometría.

Los registros fueron sometidos a un proceso de revisión y análisis para posteriormente realizar un estudio bibliométrico de tipo descriptivo, mediante la herramienta MS Excel.

Los datos fueron clasificados por producción científica anual, países, áreas temáticas y por los principales idiomas de los resultados encontrados. También se realiza un análisis referente a las revistas donde se publican los resultados obtenidos y finalmente se realiza una clasificación en cuanto a artículos que poseen un enfoque empírico y teórico.

Se muestra que en este campo de estudio la producción científica en lo referente a la Auditoria de Recursos Humanos es baja, pero que tiene una tendencia al aumento en el futuro y que esta se concentra mayormente en Latinoamérica. También se destaca el predominio de artículos de enfoque empírico por sobre los artículos de enfoque teórico.
\end{abstract}

Palabras Claves: auditoría de recursos humanos, auditoría del conocimiento, bibliometría, gestión del talento, indicadores de producción científica, recursos humanos.

Recepción: 29/09/2020. Aprobación: 22/12/2020.

1 Universidad de Talca, Facultad de Economía y Negocios, Talca, Chile, jsanchez@utalca.cl

2 Universidad de Talca, Facultad de Economía y Negocios, Talca, Chile, iyanez15@alumnos.utalca.cl 


\begin{abstract}
The purpose of this article is to carry out a review of the scientific production related to Human Resources Auditing in the SciELO database, considering it as an initial link in terms of quality compared to world class databases such as Scopus and Web of Science, using the techniques provided by bibliometrics.

The records were subjected to a process of observation and analysis to subsequently carry out a descriptive bibliometric study, using the MS Excel tool.

The data were classified by annual scientific production, countries, thematic areas and by the main languages of the results found. An analysis is also made regarding the magazines where the results obtained are published and finally a classification is made regarding articles that have an empirical and theoretical approach.

It is shown that in this field of study the scientific production in relation to the Human Resources Audit is low, but that it has a tendency to increase in the future and that it is concentrated mainly in Latin America. The predominance of articles with an empirical focus over articles with a theoretical focus is also highlighted.
\end{abstract}

Key words: bibliometrics, human resources auditing, human resources, knowledge auditing, scientific publication indicators, talent management.

\title{
INTRODUCCIÓN
}

La revisión de la literatura independiente del área de interés permite encontrar una gran variedad de investigación y producción científica. Según indica Lewison (2004), estas publicaciones ofrecen elementos claves para medir aspectos importantes de la ciencia como autores personales, direcciones institucionales, títulos de revistas, indicando no solo el país de origen de la publicación, sino también el campo de investigación y su estado del arte, citas y palabras claves. Dichas publicaciones constituyen los bloques con los que se construye la ciencia, además de una fuente inestimada de datos (Moya Anegon y Olmeda Gomez, 2010). Como consecuencia de lo anterior y dada la gran variedad de publicaciones en la investigación y producción científica, es que nace la necesidad de analizar bibliográficamente las estadísticas mediante técnicas cuantitativas. La bibliometría se centra en la realización de los distintos cálculos y en el análisis del resultado, de lo que es cuantificable en la producción y en el consumo de la información de carácter científica (González, Guzmán y Chaviano 2015).

La Gestión del Recurso Humano (en adelante GRH), está orientada a actividades de carácter operativo y administrativo, por lo que se debe proveer a la empresa de colaboradores con las competencias genéricas adecuadas y técnicas transversales (Sánchez, 2000). Con el paso del tiempo se ha perfeccionado el término de GRH, entendiendo que se está tratando con personas, por lo tanto, se asocia que los seres humanos son talentos y no recursos.

Dado lo anterior es que las teorías actuales de la gestión del talento humano ya no obedecen únicamente a las áreas de conocimiento como la sociología o la administración, sino que existen diferentes aproximaciones desde otras ciencias y disciplinas para producir una nueva filosofía teórica utilizada por la administración para abordar el sujeto en estudio, como lo son los actores dentro de la organización (Ramírez et al. 2019). 
La GRH es de suma importancia dentro de la organización, ya que sienta las bases de las acciones necesarias para lograr las metas y objetivos estratégicos que se propone la organización, frente a esto se hace indispensable un proceso de Auditoria del Recursos Humanos (en adelante $\mathrm{ARH}$ ), debido a que en las empresas los recursos de información y conocimiento mediante el incremento de las capacidades del personal, no son bien gestionados y por consiguiente no siempre existe conciencia sobre su real valor (Medina Nogueira et al. 2019). En el área de la GRH se puede encontrar un amplio universo de producción científica al respecto, pero si esta búsqueda se limita a producción científica referente a la ARH con una metodología de enfoque teórico, este universo se reduce bastante (Sánchez Pérez 2013).

Con el paso del tiempo el concepto de Capital Humano ha ido experimentando diferentes modificaciones que obedecen a nuevas filosofías que las organizaciones van adoptando conforme transcurre el tiempo y también tiene directa relación con las nuevas tendencias que va experimentando la sociedad. El concepto factor humano representa a un hombre que hace algo, devolviendo la calidad de persona humana, por lo tanto, los recursos son medios en manos del factor humano, que es quien da sentido a la realidad empresarial (Werther y Davis. 2013; de Hurtado y Leal, 2008; Chiavenato, 2013).

Dada la relevancia del concepto de la GRH dentro de las organizaciones y la sensibilidad que este tópico tiene en el contexto laboral, razón por la cual este concepto ha experimentado una serie de modificaciones a lo largo del tiempo, se realizó un análisis bibliométrico para constatar el estado de la producción científica en lo que respecta a este tema en la base de datos Scientific Electronic Library Online (SciELO). Por otra parte, la importancia de hacer este tipo de análisis que realiza una evaluación de la actividad científica y su impacto en la sociedad, radica en adecuar de forma conveniente la asignación de recursos que favorecen la investigación y el desarrollo (Sancho 1990).

Con base en lo anterior se realiza un análisis de la producción científica del tema de ARH en las bases de datos SciELO, llevando a cabo un análisis bibliométrico de las publicaciones encontradas. Esto con la finalidad de evidenciar la situación del desarrollo científico en Latinoamérica considerando a SciELO como eslabón inicial de la investigación científica en términos de calidad, para después considerar bases de datos mundiales como Scopus y Web of Science (Almeida y Gracio 2019)

En la búsqueda realizada se exportaron todos los indicadores que brindan información respecto de los resultados encontrados, para posteriormente realizar un análisis mediante las distintas técnicas que proporciona la bibliometría, por consiguiente, se puede realizar un análisis de índices como la cantidad de artículos, redes de cocitaciones y coautorías, productividad por países y por autores e impacto de las revistas (Duque y Cervantes-Cervantes, 2019).

El objetivo de la presente investigación es realizar una revisión sistemática mediante las técnicas que proporciona la bibliometría y realizar un análisis de la producción científica sobre ARH. 


\section{METODOLOGÍA, MATERIALES Y MÉTODOS}

Se realizó un estudio bibliométrico descriptivo de la producción científica referente a la ARH, con la finalidad de recoger información de manera independiente o conjunta sobre los conceptos o las variables a las que se refieren (Hernández Sampieri 2014). La búsqueda se desarrolló en la base de datos SciELO, la que actualmente cuenta con más de mil revistas indexadas activas. Se considera esta base de datos con el objetivo de evidenciar la situación de la producción científica a nivel latinoamericano y por el prestigio, en términos de calidad, que posee dentro de esta región geográfica (Almeida y Gracio 2019; Castrillón-Estrada et al. 2008).

Se implementó la ecuación de búsqueda (Knowledge Auditing) OR (Auditoria del Conocimiento) OR (Human Resources Auditing) OR (Auditoria de Recursos Humanos) OR (Labour Auditing) OR (Auditoria Laboral), la que fue el resultado final de una serie de ecuaciones de búsqueda que fueron sometidas a filtros con base en los resultados obtenidos. De la búsqueda realizada se obtuvo un total final de 47 documentos, los cuales abordan el tema de la ARH, en los cuales se basa el análisis cualitativo de esta investigación (tabla 1).

\section{Tabla 1}

Resultados de búsqueda sobre ARH en la base de datos SciELO

\begin{tabular}{lc}
\hline \multicolumn{1}{c}{ Ecuación de búsqueda } & Resultados \\
\hline Human Resources & 4.283 \\
Human Resources Management & 1.279 \\
$\begin{array}{l}\text { (Human Resources Management) OR (Auditing) } \\
\text { (Human Talent Management) OR (Auditing) }\end{array}$ & 1.532 \\
$\begin{array}{l}\text { Knowledge Audit } \\
\text { (Knowledge auditing) OR (auditoria del conocimiento) OR (human resources au- } \\
\begin{array}{l}\text { diting) OR (auditoria de recursos humanos) OR (labour auditing) OR (auditoria } \\
\text { laboral) OR (human talent auditing) OR (auditoria del talento humano) }\end{array}\end{array}$ & 343 \\
\hline
\end{tabular}

Fuente: Elaboración propia con base en datos de SciELO (2020).

Los resultados obtenidos fueron sometidos a un análisis mediante la herramienta MS Excel, debido a que posee las funcionalidades para realizar un análisis estadístico exhaustivo y al mismo tiempo permite visualizar esta información a través de la elaboración de tablas y gráficos. Mediante esta herramienta se midió la producción anual en la base de datos de SciELO, la producción por países, lo que derivó a realizar un análisis de los idiomas presentes en los documentos encontrados y también se clasificó según las áreas temáticas propuestas por la base de datos de SciELO. También fueron analizadas las fuentes en que se publican los documentos que tratan sobre el tema de ARH, clasificándolo en base a las revistas indexadas y finalmente se desarrolló un análisis de los documentos, determinando si estos seguían un enfoque empírico o teórico. 
No se estableció un rango de tiempo específico para realizar esta búsqueda, ya que, de esta manera, el número total de resultados refleja toda la producción científica desde sus inicios hasta la actualidad en el campo de ARH y otras áreas que tienen directa relación con esta en la base de datos SciELO.

\section{RESULTADOS}

La base de datos SciELO que a la fecha cuenta con más de mil revistas indexadas activas, permite realizar una búsqueda exhaustiva sobre el tópico de ARH.

Utilizando la ecuación de búsqueda (Knowledge Auditing) OR (Auditoria del Conocimiento) OR (Human Resources Auditing) OR (Auditoria de Recursos Humanos) OR (Labour Auditing) OR (Auditoria Laboral) OR (Human Talent Auditing) OR (Auditoria del Talento Humano) en el buscador de la base de datos, esta arroja un total de 47 artículos relacionados principalmente a la ARH. Cabe destacar que la búsqueda se debe realizar en el idioma inglés, ya que al realizarla únicamente en el idioma español esta arroja solo 33 resultados.

$\mathrm{Al}$ realizar un análisis con respecto a las publicaciones existentes en la base de datos de SciELO filtrándolas por la fecha de publicación entre los periodos comprendidos entre los años 2003 y 2020, se puede apreciar que se refleja una tendencia al aumento de la producción científica conforme pasa el tiempo (figura 1).

\section{Figura 1}

\section{Producción científica anual sobre ARH}

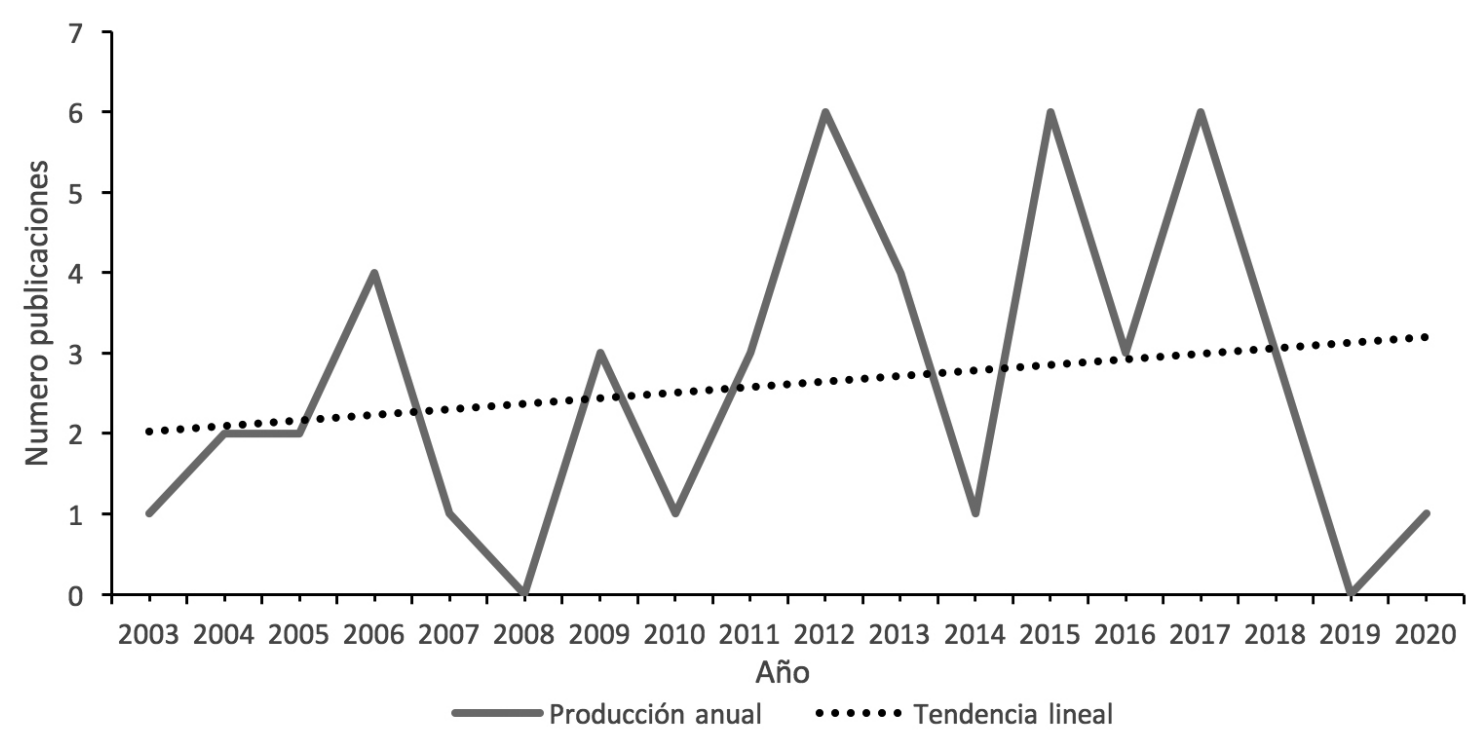

Fuente: Elaboración propia con base en datos de SciELO (2020). 
Dada la búsqueda realizada, se pudo apreciar que el primer artículo sobre ARH en esta base de datos, data del año 2003, siendo hasta el año 2010 el rango de menor productividad en cuanto a elaboración de artículos científicos, publicando solo 14 documentos. Desde el año 2011 en adelante se aprecia una clara tendencia al aumento de la producción científica, siendo hasta el año 2018 el período de mayor productividad, en donde se refleja el 68\% del total de la producción científica en estudio.

Se apreció que en el año 2012 se registra el primer récord de publicaciones, sumando 6 documentos, para el año 2013 nuevamente disminuye hasta el año 2015, donde iguala el récord antes señalado, hecho que también se repite el año 2017. Para los años posteriores nuevamente la producción baja, siendo en el año 2020 donde la producción científica retoma el camino del aumento, encontrando un artículo para dicho año. En base al análisis detallado de documentos, se pudo apreciar que en las recomendaciones de los autores, se planteaba la necesidad de seguir investigando en el área.

En lo que se refiere a la producción por países (figura 2) se puede constatar que Colombia lidera en cuanto a producción científica en lo referente a ARH, sumando un total de 11 artículos, lo cual representa un $23 \%$ del total de la producción científica en el área ya mencionada. El segundo país que más produce documentos en el área es Brasil que totaliza 9 artículos, sigue Cuba con 8 publicaciones, posteriormente se ubican México y Sudáfrica sumando 4 resultados cada uno, luego están Chile, España y Uruguay con 2 resultados cada uno y finalmente se ubican Argentina, Bolivia, Portugal, Venezuela y Ecuador, con una publicación cada uno. Como se puede visualizar, América del Sur lidera la lista de producción por países (Colombia y Brasil) en el tema de ARH en la base de datos de SciELO comparado con países de América del Norte (Cuba y México), representando un $60 \%$ del total de artículos encontrados, esto debido a que el repositorio de esta base de datos se compone principalmente por revistas provenientes de esta región geográfica. Al mismo tiempo esto representa un dato no menor que refleja el importante rol que cumple Sudamérica en el desarrollo investigativo en esta área.

\section{Figura 2}

\section{Producción científica sobre ARH por países}

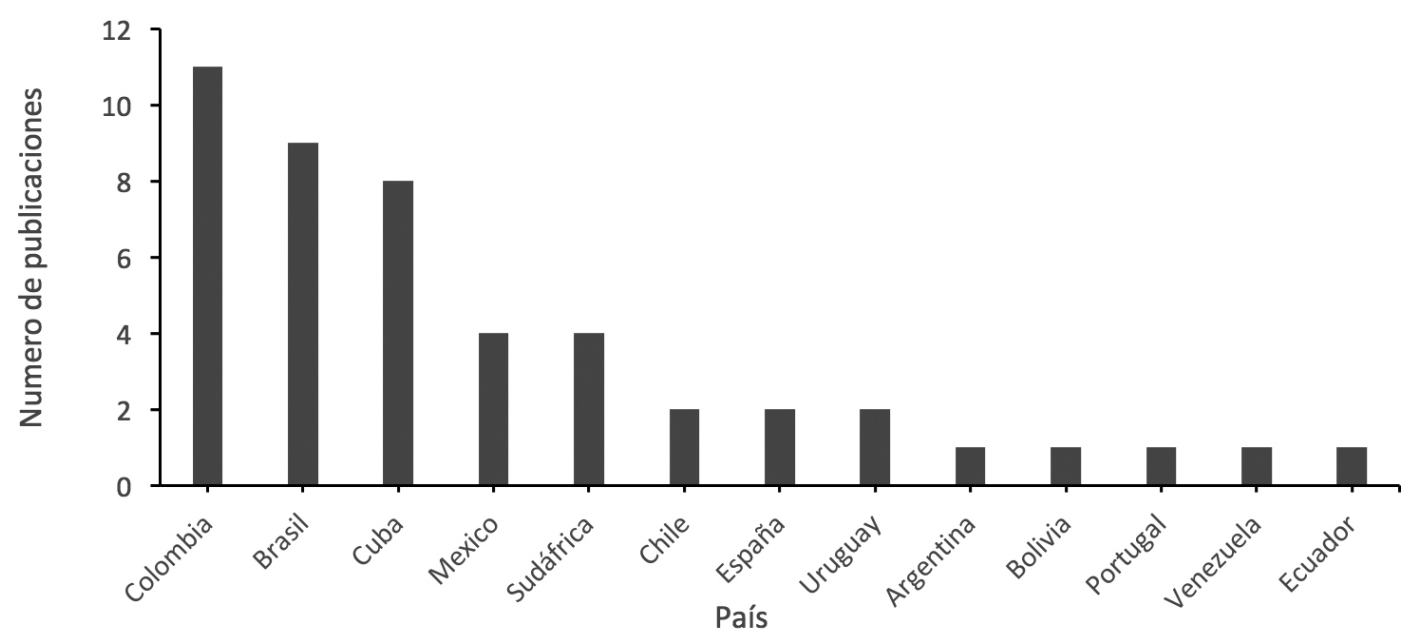

Fuente: Elaboración propia con base en datos de SciELO (2020). 
En cuanto al idioma de los resultados encontrados (tabla 2), estos tienen un comportamiento inusual, ya que el idioma español domina en la producción científica en estudio con 24 resultados, representando un $64 \%$ del total de la producción científica, lo cual no deja de ser relevante, ya que al incluir en la ecuación de búsqueda términos en inglés, la tendencia pudo haber estado inclinada a encontrar más resultados en ese idioma, ya que se le considera el idioma universal de la ciencia, haciendo un espacio en publicaciones de alta visibilidad internacional (Dorta Contreras 2006). El idioma inglés solo reportó 10 resultados, posteriormente se encuentra la producción científica en idioma portugués, con 8 resultados. De esta manera se registró un resultado completamente opuesto a lo esperado, ya que existen incentivos para los autores que publican en idioma inglés, de manera que obtienen un mayor nivel de difusión y reconocimiento.

\section{Tabla 2}

\section{Producción científica por idioma}

\begin{tabular}{lc}
\hline \multicolumn{1}{c}{ Idioma } & Numero de resultados \\
\hline Español & 32 \\
Inglés & 10 \\
Portugués & 8 \\
\hline
\end{tabular}

Nota. La suma de los resultados encontrados es de 50, distinto al total antes señalado, ya que existen 3 documentos que se encuentran en 2 idiomas, inglés y portugués. Fuente: Elaboración propia con base en datos de SciELO (2020).

En la clasificación de los resultados de la búsqueda por área temática (figura 3), la base de datos SciELO clasificó los resultados en áreas como Ciencias Sociales Aplicadas (Applied Social Sciences), Ciencias de la Salud (Health Sciences), Ciencias Biológicas (Biological Sciences), Ciencias Humanas (Human Sciences), Ingeniería (Engineering) y un área Multidisciplinaria (Multidisciplinary).

Se pudo apreciar que las principales áreas de estudio en esta base de datos fueron ciencias de la salud, representando un $40 \%$ de la búsqueda, seguido por ciencias sociales aplicadas, la cual representó un 37\%, un $9 \%$ perteneciente al área de ciencias biológicas y en la misma proporción para el área de ingeniería. Es importante señalar que por regla general algunos artículos pueden encasillarse en más de una categoría. 


\section{Figura 3}

Producción científica por área temática en SciELO

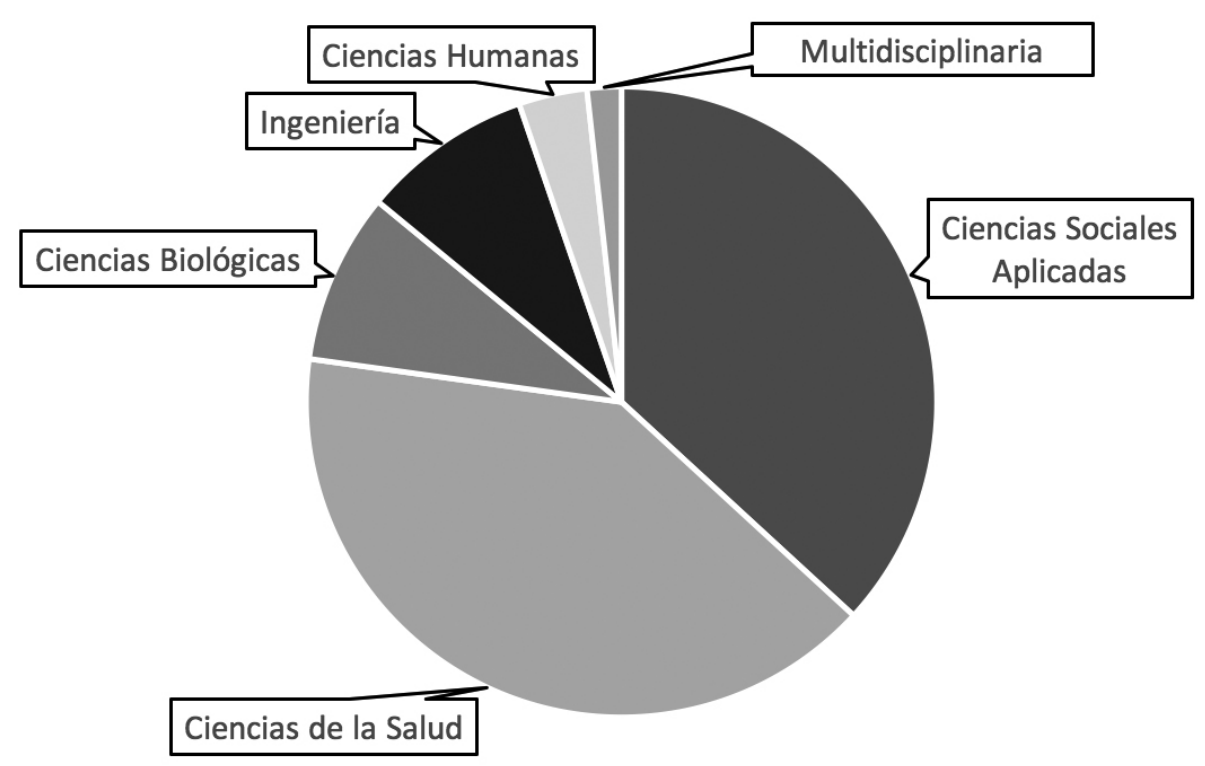

Fuente: Elaboración propia con base en datos de SciELO (2020).

Realizando un análisis a las fuentes donde se publicaron los resultados de la búsqueda sobre el tópico de $\mathrm{ARH}$, se encuentra una gran cantidad de revistas indexadas en la base de datos SciELO (40 revistas), las cuales se enmarcan en distintas áreas temáticas tales como, ciencias sociales aplicadas, ciencias de la salud, ciencias biológicas, ingeniería, ciencias humanas y áreas multidisciplinarias. Se constató que el índice de publicación de artículos por revista es bajo, el cual no supera el $5 \%$ en el caso de las revistas que tienen más publicaciones ( 2 documentos). La situación se explica en el hecho de que el total de artículos publicados no se concentra en gran cantidad en solo algunas revistas, se distribuyen de una forma más equitativa, aportando una cantidad similar de artículos científicos sobre ARH, existiendo un grupo de 7 revistas, las cuales poseen 2 artículos cada una y otro grupo de 33 revistas, que aportan un artículo cada una. Aplicando el promedio como medida de tendencia central, el cual representa el punto de equilibrio de la distribución de los datos (Rendón-Macías et al., 2016) arroja un total de 1,2 artículos publicados por revista aproximadamente. 
Tabla 3

Revistas donde se publica producción científica de ARH

\begin{tabular}{|c|c|c|}
\hline Revista & $\mathrm{N}$ & $\%$ \\
\hline Archivos de Pediatría del Uruguay & 2 & $4 \%$ \\
\hline Contaduría y administración & 2 & $4 \%$ \\
\hline Cuadernos de Contabilidad & 2 & $4 \%$ \\
\hline Curationis & 2 & $4 \%$ \\
\hline Estudios Gerenciales & 2 & $4 \%$ \\
\hline Revista Cubana de Información en Ciencias de la Salud & 2 & $4 \%$ \\
\hline Revista da Escola de Enfermagem da USP & 2 & $4 \%$ \\
\hline ACIMED & 1 & $2 \%$ \\
\hline Biosalud & 1 & $2 \%$ \\
\hline Educación Médica Superior & 1 & $2 \%$ \\
\hline Enfoque UTE & 1 & $2 \%$ \\
\hline Escritos de Psicología (Internet) & 1 & $2 \%$ \\
\hline $\begin{array}{l}\text { Fides et Ratio - Revista de Difusión cultural y científica de la Universidad La } \\
\text { Salle en Bolivia }\end{array}$ & 1 & $2 \%$ \\
\hline Formación universitaria & 1 & $2 \%$ \\
\hline Gaceta Sanitaria & 1 & $2 \%$ \\
\hline Información, cultura y sociedad & 1 & $2 \%$ \\
\hline Ingeniería Industrial & 1 & $2 \%$ \\
\hline Innovar & 1 & $2 \%$ \\
\hline Nova Economia & 1 & $2 \%$ \\
\hline Nova scientia & 1 & $2 \%$ \\
\hline Pensamiento y Gestión & 1 & $2 \%$ \\
\hline RAM. Revista de Administração Mackenzie & 1 & $2 \%$ \\
\hline RISTI - Revista Ibérica de Sistemas e Tecnologias de Informação & 1 & $2 \%$ \\
\hline Revista Brasileira de Enfermagem & 1 & $2 \%$ \\
\hline Revista Brasileira de Epidemiologia & 1 & $2 \%$ \\
\hline Revista Contabilidade y Finanças & 1 & $2 \%$ \\
\hline Revista Cubana de Medicina Tropical & 1 & $2 \%$ \\
\hline Revista Facultad Nacional de Salud Pública & 1 & $2 \%$ \\
\hline Revista Médica Electrónica & 1 & $2 \%$ \\
\hline Revista Universidad y Sociedad & 1 & $2 \%$ \\
\hline Revista chilena de infectología & 1 & $2 \%$ \\
\hline
\end{tabular}




\begin{tabular}{|c|c|c|}
\hline Revista & $\mathrm{N}$ & $\%$ \\
\hline Revista de Ciencias Sociales & 1 & $2 \%$ \\
\hline Revista de Ingeniería & 1 & $2 \%$ \\
\hline Revista de Salud Pública & 1 & $2 \%$ \\
\hline Revista de Saúde Pública & 1 & $2 \%$ \\
\hline Revista republicana & 1 & $2 \%$ \\
\hline SAMJ: South African Medical Journal & 1 & $2 \%$ \\
\hline South African Journal of Occupational Therapy & 1 & $2 \%$ \\
\hline Textual: análisis del medio rural latinoamericano & 1 & $2 \%$ \\
\hline Transinformação & 1 & $2 \%$ \\
\hline Total & 47 & $100 \%$ \\
\hline
\end{tabular}

Fuente: Elaboración propia con base en datos de SciELO (2020).

Dentro de los artículos que fueron analizados se apreció que estos se enmarcaron, por un lado, en un enfoque empírico, lo cual hace referencia a la elaboración de instrumentos que comprueben el comportamiento de un determinado grupo de interés, mediante el procesamiento de datos verídicos y comprobables. Por otro lado, se destacó la existencia de documentos que se enmarcaron en un enfoque teórico, los cuales describen detalladamente un tema de interés desde una perspectiva concreta, elaborando teorías que van generando conocimiento con respecto al tema que se está desarrollando.

Se constató la predominancia de documentos basados en el enfoque empírico, representando una amplia mayoría con respecto del número de documentos sujetos a estudio, existiendo un total de 32 artículos ocupando este enfoque, lo que representó un $68 \%$ del total de la producción científica, mientras que en el enfoque teórico existe un total de 15 artículos, que representaron un $32 \%$ del total (figura 4 ). 


\section{Figura 4}

Análisis de resultados en base a enfoque Empírico y Teórico

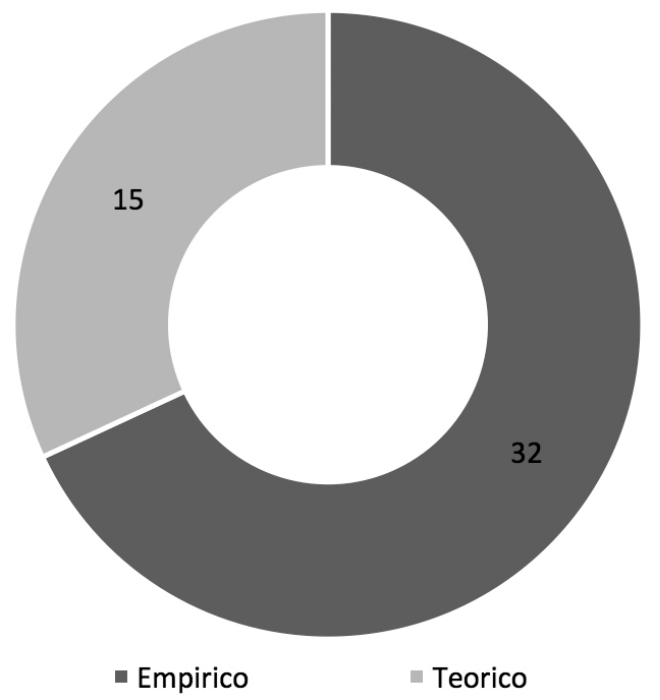

Nota. Fuente: Elaboración propia con base en datos de SciELO (2020).

De los documentos recientes que fueron sometidos a análisis que contaban con un enfoque teórico (últimos 3 años) destacan: Parra Osorio et al. (2018) quienes realizaron un análisis/contraste utilizando documentos originales occidentales y textos históricos, analizando la salud ocupacional como medicina laboral, higiene ocupacional, seguridad industrial y auditoria. También Galvis-Castañeda y Santos-Mera (2017) quienes llevaron a cabo un estudio sobre el fenómeno del fraude desde la perspectiva del estafador que interesa a disciplinas como la criminología, contabilidad, auditoria y psicología, explicándolo mediante las variables presión, oportunidad y racionalización. Ramírez Ospina y Andrade Arango (2017)social and environmental realizaron un estudio el que trató sobre el aporte de la contabilidad y la auditoria, destacando la importancia de estos conceptos en la relación del ser humano con la naturaleza. Por su parte Proaño Escalante et al. (2017) brindó una caracterización y uso de los sistemas que se basan en conocimiento aplicado a la auditoria de sistemas de información, con el objetivo de ayudar al auditor informático cuando emite una opinión sobre un hallazgo en un área determinada.

\section{DISCUSION Y CONCLUSIONES}

La investigación abordó la evolución y producción científica en la base de datos de SciELO durante los últimos 18 años, mediante la utilización de indicadores bibliométricos.

El principal factor que determina la necesidad de realizar esta investigación es la poca producción científica en el área de ARH usando un enfoque teórico, lo cual queda demostrado, específicamente en la base de datos de SciELO, ya que esta solo representó un 32\% del total de la población en estudio. Siendo además, solo un artículo el cual trata directamente sobre dicho tópico, 
perteneciente a González Guitián y Ponjuán Dante (2016) donde se realiza un estudio reflexivo en cuanto a metodologías, modelos y métodos para auditar el conocimiento en las organizaciones actuales.

Efectivamente existe una baja cantidad de producción científica en general en este tema, pero según la tendencia que se puede apreciar una vez procesados los resultados en la base de datos de MS Excel, es que esta tendería a aumentar conforme pasa el tiempo, si es que todo se mantiene constante.

La predominancia del idioma español fue una constante en esta investigación, esto principalmente porque la base de datos se compone de revistas iberoamericanas. Además se estima que los autores con mayor habilidades en lo referente al dominio de otras lenguas tienden a publicar en revistas anglófonas (Tomás-Casterá, Sanz-Valero, y Juan-Quilis 2013). Otro punto por el cual se puede explicar es que la producción científica en estudio se concentró en un $70 \%$ en países en que su idioma nativo es el español.

Como limitaciones para realizar esta investigación se puede decir que la interfaz de la base de datos SciELO, dificulta en gran medida el cálculo de más indicadores bibliométricos, razón por la cual no se pudo generar estadística relacionada a los niveles de citaciones, cocitaciones, entre otros, debido a que la plataforma SciELO Analytics a la fecha, se encuentra en una etapa de desarrollo.

Según la clasificación que entrega la base de datos, se puede notar que no existen áreas temáticas que hagan mención específica sobre negocios, contabilidad, administración o áreas afines, lo cual no significa que no existan estudios asociados al caso. Claro es el ejemplo del articulo The perceptions of midwives regarding audit and feedback on the use of the partogram at Vhembe District of Limpopo Province, South Africa, en el que se tratan las percepciones del personal clínico sobre el proceso de auditoría del partograma por parte de los profesionales de salud (Lumadi 2017), el cual se encasilla dentro de un área médica, pero hace clara referencia a un proceso de ARH en un área de trabajo especifica.

Para la realización de estudios bibliométricos en el futuro, se recomienda ampliar el alcance del trabajo a las bases de datos Scopus (Elsevier) o Web of Science (Clarivate), para así tener una visión más global del aporte de la bibliometria a la auditoría de recursos humanos. 


\section{REFERENCIAS BIBLIOGRÁFICAS}

Almeida, Catia Candida, y Maria Claudia Cabrini Gracio. 2019. “Produção científica brasileira sobre o indicador 'Fator de Impacto': um estudo nas bases SciELO, Scopus e Web of Science". Encontros Bibli: revista eletrônica de biblioteconomia e ciência da informação 24(54):62-77.

Castrillón-Estrada, Jaime A., Juan Camilo García Domínguez, Marco Anaya Taboada, Deisy Rodríguez Berdugo, Dereck De la Rosa Barranco, y Carlo V Caballero-Uribe. 2008. “Bases de datos, motores de búsqueda e índices temáticos: herramientas fundamentales para el ejercicio médico". Salud Uninorte 24(1): 95-119.

Chiavenato, I. (2017). Administración de recursos humanos. El capital humano de las organizaciones (10 Edición). España: McGraw Hill Interamericana.

Dorta Contreras, C. Alberto Juan. 2006. "En defensa de nuestra producción científica”. ACIMED 14(3):0-0.

Duque, Pedro, y Luis Salvador Cervantes-Cervantes. 2019. “University social responsibility: A systematic review and a bibliometric analysis". Estudios Gerenciales 35(153): 451-64.

Galvis-Castañeda, Iván Eduardo, y Jaime Eduardo Santos-Mera. 2017. “Geometría del fraude TT - Geometry of fraud TT - Geometria da fraude". Cuadernos de Contabilidad 18(45): 74-85.

González Guitián, María Virginia, y Gloria Ponjuán Dante. 2016. “Metodologías y modelos para auditar el conocimiento: análisis reflexivo". Información, cultura y sociedad (35): 65-90.

González, María Josefa Peralta, Maylín Frías Guzmán, y Orlando Gregorio Chaviano. 2015. “Criterios, clasificaciones y tendencias de los indicadores bibliométricos en la evaluación de la ciencia". Revista Cubana de Información en Ciencias de la Salud (ACIMED) 26(3): 290-309.

Hernández Sampieri, Roberto. 2014. Metodología de la investigación. 6a. ed. editado por C. Fernández Collado y M. del P. Baptista Lucio. México: México: McGraw-Hill.

de Hurtado, María C. Garcáa, y Martín Leal. 2008. “Evolución histórica del factor humano en las organizaciones: de recurso humano a capital intelectual". Omnia 14(3): 144-59.

Lewison, Grant. 2004. "Researchers" and Users" Perceptions of the Relative Standing of Biomedical Papers in Different Journals". Scientometrics 53(2): 229-40.

Lumadi, Thanyani G. 2017. "The Perceptions of Midwives Regarding Audit and Feedback on the Use of the Partogram at Vhembe District of Limpopo Province, South Africa". Curationis 40(1): 1-6.

Medina Nogueira, Yuly Esther, Yusef El Assafiri Ojeda, Alberto Medina León, Dianelys Nogueira Rivera, y Daylin Medina Nogueira. 2019. "La auditoría del conocimiento como herramienta de apoyo a la gestión universitaria". Conrado 15(69): 324-33.

Moya Anegon, Felix de, y Carlos Olmeda Gomez. 2010. Indicadores bibliometricos de la actividad cientifica de Cataluna. 2010. Editorial UOC.

Parra Osorio, Liliana, Floro Hermes Gómez Pineda, Erick Lanny Rojas Parra, Martín Acosta-Fernández, y Teresa Margarita Torres-López. 2018. “SINDÉRESIS HERMENÉUTICA DE LA LEY 1562 Y EL CONCEPTO DE LA SALUD OCUPACIONAL TT - Hermeneutical understanding of the Act 1562 and the concept of occupational health". Revista republicana (24): 21-40.

Proaño Escalante, Rodrigo Arturo, Ciro Napoleón Saguay Chafla, Segundo Bolívar Jácome Canchig, y Fanny Sandoval Zambrano. 2017. "Sistemas basados en conocimiento como herramienta de ayuda en la auditoría de sistemas de información TT - Knowledge based systems as an aid in information systems audit". Enfoque UTE 8: 148-59. 
Ramírez Ospina, Duván Emilio, y Yamilhet Andrade Arango. 2017. “Aporte de la contabilidad y la auditoría desde una perspectiva ambiental TT - Contribution of accounting and auditing from an environmental perspective". Textual: análisis del medio rural latinoamericano (69): 27-41.

Ramírez, Reynier I., Cesar A. Espindola, Gladis I. Ruíz, y Alfredo M. Hugueth. 2019. “Gestión del Talento Humano: Análisis desde el Enfoque Estratégico TT - Human Talent Management: Analysis from the Strategic Approach". Información tecnológica 30(6): 167-76.

Rendón-Macías, Mario Enrique, Miguel Ángel Villasís-Keeve, y María Guadalupe Miranda-Novales. 2016. “Estadística descriptiva”. Revista Alergia México 63(4): 397-407.

Sánchez Henríquez, Jorge. 2000. Auditoría de la Formación. Revista Forum Empresarial. (5): 52-72.

Sánchez Pérez, José. 2013. Fundamentos de auditoría de recursos humanos: 101 indicadores clave. McGraw-Hill España.

Sancho, Rosa. 1990. “Indicadores bibliométricos utilizados en la evaluación de la ciencia y la tecnología. Revisión bibliográfica". Revista española de documentación científica 13(3-4).

Tomás-Casterá, Vicente, Javier Sanz-Valero, y Verónica Juan-Quilis. 2013. “Estudio bibliométrico de la producción científica y de consumo de las revistas sobre nutrición indizadas en la red SciELO". Nutricion Hospitalaria 28(3): 969-70.

Werther, W. y Davis, K. 2013. Administración de recursos humanos, el capital humano de las empresas (7 $7^{a}$ Edición). México: McGraw-Hill Interamericana.

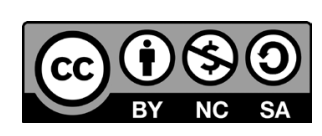

Esta obra está bajo una licencia de

Creative Commons Atribución-NoComercial-CompartirIgual 4.0 Internacional 